\title{
Cluster Analysis of Malware Family Relationships
}

\author{
Samanvitha Basole* Mark Stamp ${ }^{\dagger, \text { "ै }}$
}

\begin{abstract}
In this paper, we use $K$-means clustering to analyze various relationships between malware samples. We consider a dataset comprising 20 malware families with 1000 samples per family. These families can be categorized into seven different types of malware. We perform clustering based on pairs of families and use the results to determine relationships between families. We perform a similar cluster analysis based on malware type. Our results indicate that $K$-means clustering can be a powerful tool for data exploration of malware family relationships.
\end{abstract}

\section{Introduction}

Previous research has demonstrated that it is possible in some cases to train a machine learning model to detect multiple malware families [3]. Specifically, neighborhood-based techniques are relatively effective in such a situation. Although support vector machines (SVM) did not perform well in this previous research, both $k$-nearest neighbors $(k-\mathrm{NN})$ and random forest $(\mathrm{RF})$ were able to distinguish malware from benign with good accuracy, even when several malware families were combined to form the malware class.

In this research, we consider the same dataset used in [3], which includes 20 malware families. Here, we apply cluster analysis to these families. Our goal is to determine whether we can discover interesting connections, relationships, and differences between these various families, based on elementary clustering techniques. While [3] considers binary classification experiments to distinguish malware from benign, the research in this paper is focused on a data exploration problem. The features we use are byte $n$-gram frequencies, while the clustering method we consider is the well-known $K$-means algorithm.

The remainder of this paper is organized as follows. In Section 2, we provide relevant background information, including a brief discussion of related work. Then in Section 3, we give our experimental results and analysis. Finally, Section 4 concludes this paper, where we have included suggestions for future work.

\section{Background}

In this section, we first consider relevant related work. Then we discuss our malware dataset, and we provide information on each family in the dataset. We also present the various metrics that we use in our clustering experiments. Finally, we provide an introduction to the $K$-means clustering algorithm.

\section{$2.1 \quad$ Related Work}

In [9], the authors use API calls to classify malware based on their types. They consider a random forest $(\mathrm{RF})$ classifier and achieve an average area under the ROC curve (AUC) of 0.98. In contrast, we use $n$-grams and a clustering approach, and we are considering a data exploration problem, rather than a straightforward classification problem.

The authors in [4] propose a clustering approach for malware, wherein the goal is to cluster samples based on similar behavior. These authors use features such as system calls and network activity, to cluster malicious code based on its behavior. In contrast to this previous work, our cluster analysis is based on simpler and easier to collect features and, again, we are in a data exploration mode of operation.

The research in [10] uses BIRCH clustering based on static and dynamic features. These authors consider 18 families, but 12 of those families contain less than 100 samples each. Our approach uses a larger and balanced dataset for clustering.

\footnotetext{
*s97basole@gmail.com

${ }^{\dagger}$ mark.stamp@sjsu.edu

"Department of Computer Science, San Jose State University, San Jose, California
} 


\subsection{Dataset}

The same dataset as used in [3] is considered in this research. This dataset includes 20 families, which we categorize into malware types, as listed in Table 1.

Table 1: Type of each malware family

\begin{tabular}{ccc|ccc}
\hline \hline Index & Family & Type & Index & Family & Type \\
\hline 0 & Adload [18] & Trojan Downloader & 10 & Obfuscator [27] & VirTool \\
1 & Agent [19] & Trojan & 11 & OnLineGames [13] & Password Stealer \\
2 & Alureon [25] & Trojan & 12 & Rbot [28] & Backdoor \\
3 & BHO [21] & Trojan & 13 & Renos [20] & Trojan Downloader \\
4 & CeeInject [24] & VirTool & 14 & Startpage [22] & Trojan \\
5 & Cycbot.G [2] & Backdoor & 15 & Vobfus [29] & Worm \\
6 & DelfInject [11] & VirTool & 16 & Vundo [30] & Trojan Downloader \\
7 & FakeRean [26] & Rogue & 17 & Winwebsec [31] & Rogue \\
8 & Hotbar [1] & Adware & 18 & Zbot [14] & Password Stealer \\
9 & Lolyda.BF [12] & Password Stealer & 19 & Zeroaccess [23] & Trojan \\
\hline \hline
\end{tabular}

Each of the malware families in Table 1 is summarized below.

Adload downloads an executable file, stores it remotely, executes the file, and disables proxy settings [18].

Agent downloads Trojans or other software from a remote server [19].

Alureon exfiltrates usernames, passwords, credit card data, and other confidential data from an infected system [25].

BHO can perform a variety of actions, guided by an attacker [21].

CeeInject uses advanced obfuscation to avoid being detected by antivirus software [24].

Cycbot.G connects to a remote server, exploits vulnerabilities, and spreads through backdoor ports [2].

DelfInject sends usernames, passwords, and other personal and private information to an attacker [11].

FakeRean pretends to scan the system, notifies the user of supposed issues, and asks the user to pay to clean the system [26].

Hotbar is adware that shows ads on webpages and installs additional adware [1].

Lolyda.BF sends information from an infected computer and monitors the system. It can share user credentials and network activity with an attacker [12].

Obfuscator tries to obfuscate or hide itself to defeat malware detectors [27].

OnLineGames steals login information of online games and tracks user keystroke activity [13].

Rbot gives control to attackers via a backdoor that can be used to access information or launch attacks, and serves as a gateway to infect additional sites [28].

Renos downloads software that claims the system has spyware and asks for a payment to remove the nonexistent spyware [20].

Startpage changes the default browser homepage and may perform other malicious activities [22].

Vobfus is a worm that downloads malware and spreads through USB drives or other removable devices [29].

Vundo displays pop-up ads and may download files. It uses advanced techniques to defeat detection [30].

Winwebsec displays alerts that ask the user for money to fix supposed issues [31].

Zbot is installed through email and shares a user's personal information with attackers. In addition, Zbot can disable a firewall [14].

Zeroaccess is a Trojan horse that downloads applications that click on ads, thereby making money for the attacker [23]. 
The features we use for clustering are based on byte $n$-gram frequencies. Specifically, we choose the top 20 byte $n$-grams, with $n=2$, from our malware class. These frequency vectors are then normalized, so that each vector can be viewed as a discrete probability distribution. The resulting normalized bigram frequency vectors (of length 20) form our feature set.

Our experiments include clustering based on pairs of families, clustering selected families belonging to different malware types, and clustering families belonging to the same malware type. The number of samples of each of the seven malware types found in our dataset is given in Table 2. Note that we categorize "Trojan Downloader" as a type of Trojan, giving us the seven distinct types listed in Table 2.

Table 2: Number of samples of each type

\begin{tabular}{cc}
\hline \hline Malware Type & Samples \\
\hline VirTool & 3000 \\
Password Stealer & 3000 \\
Backdoor & 2000 \\
Trojan & 8000 \\
Worm & 1000 \\
Rogue & 2000 \\
Adware & 1000 \\
\hline \hline
\end{tabular}

\subsection{Metrics}

In this section, we discuss the metrics used to numerically evaluate our clustering results. Note that we do not use accuracy, due to the label-switching problem that occurs when we attempt to apply this metric to clustering results [17].

One popular choice for clustering is the so-called $v$-measure, which is a robust metric for cluster evaluation-robust in the sense that a permutation of the cluster labels does not affect the score. The $v$-measure is defined as the harmonic mean between homogeneity (i.e., the case where each cluster contains all points from a single class) and completeness (i.e., the case where all points from the same class are in one cluster) [8]. Another nice feature of this metric is that it is symmetric, in that it yields the same score if the predicted classes and the true classes are switched. The $v$-measure ranges from 0 to 1 .

Although $v$-measure is a robust evaluation metric with many advantages, it is not the best choice for the research considered in this paper. The $v$-measure is not normalized for random cluster results, hence it would tend to produce a higher score for random cluster assignments when a "large" number of clusters $K$ is chosen, say, $K>10$. In contrast, adjusted mutual information (AMI) results in random label assignments having a score close to 0 , regardless of the size of the dataset or the number of clusters.

Another useful metric for clustering is the adjusted Rand index (ARI). The ARI is similar to AMI, in the sense that it is adjusted to account for chance. The Rand index is a similarity measure that considers all pairs of samples and uses the number of pairwise agreements in the true and predicted clusters. Specifically, the Rand index is calculated as [16]

$$
\mathrm{RI}=\frac{a+d}{a+b+c+d}=\frac{a+d}{\left(\begin{array}{l}
n \\
2
\end{array}\right)}
$$

where $a, b, c$, and $d$ are defined as follows: If $U$ and $V$ are two different partitions or clusterings of the same data, then let $a, b, c$, and $d$ be the number of objects determined by

$$
\begin{aligned}
& a=\text { in the same cluster in } U \text { and in the same cluster in } V \\
& b=\text { in the same cluster in } U \text { but in different clusters in } V \\
& c=\text { in the same cluster in } V \text { but in different clusters in } U \\
& d=\text { in different clusters in } U \text { and in different clusters in } V
\end{aligned}
$$


The formula for ARI is calculated using the raw Rand index RI as [8]

$$
\mathrm{ARI}=\frac{\mathrm{RI}-\mathrm{E}(\mathrm{RI})}{\max (\mathrm{RI})-\mathrm{E}(\mathrm{RI})}
$$

where $E$ is the expected value operator.

The authors in [15] state that AMI should be used when the true clusters are unbalanced in size, while ARI should be used when the true clusters are large and roughly equal-sized. In our research, the size of the ground truth for family labels is precisely balanced with 1000 samples in each family. Thus, we use ARI to evaluate our clustering predictions.

\section{$2.4 \quad K$-Means}

In this section, we first discuss a generic approach to clustering. We then consider how to implement such an approach, which leads directly to the $K$-means algorithm.

Suppose that we are given the $n$ data points $X_{1}, X_{2}, \ldots, X_{n}$, where each of the $X_{i}$ is a vector of $m$ real numbers. For example, we could analyze a set of malware samples based on, say, five distinct scores, denoted $s_{1}, s_{2}, \ldots, s_{5}$. Then each data point would be of the form

$$
X_{i}=\left(s_{1}, s_{2}, s_{3}, \ldots, s_{5}\right) .
$$

We assume that the desired number of clusters $K$ is specified in advance and that we want to partition our $n$ data points $X_{1}, X_{2}, \ldots, X_{n}$ into $K$ clusters. We also assume that we have a distance function $d\left(X_{i}, X_{j}\right)$ that is defined for all pairs of data points.

We associate a centroid with each cluster, where the centroid can be viewed as representative of its cluster. Intuitively, a centroid is the center of mass of a cluster. We denote cluster $j$ as $C_{j}$ with the corresponding centroid denoted as $c_{j}$. Note that in $K$-means, centroids need not be actual data points.

Now, suppose that we have clustered our $n$ data points. Then we have a set of $K$ centroids,

$$
c_{1}, c_{2}, c_{3}, \ldots, c_{K}
$$

and each data point is associated with exactly one centroid. Let centroid $\left(X_{i}\right)$ denote the (unique) centroid of the cluster to which $X_{i}$ belongs. The centroids determine the clusters, in the sense that whenever we have

$$
c_{j}=\operatorname{centroid}\left(X_{i}\right) \text {, }
$$

then $X_{i}$ belongs to cluster $C_{j}$.

Before we can cluster data based on the process outlined above, we need to address the following two questions.

1. How do we determine the centroids $c_{j}$ ?

2. How do we determine the clusters? That is, we need to specify the function centroid $\left(X_{i}\right)$, which assigns data points to centroids. This has the effect of determining the clusters.

There are many reasonable ways to answer these questions. Next, we consider an intuitively appealing approach that leads directly to the $K$-means algorithm.

Intuitively, it seems clear that the more "compact" a cluster is, the better. Of course, this will depend on the data points $X_{i}$ and the number of clusters $K$. Since the data is given, and we are assuming that $K$ has been specified, we have no control over the $X_{i}$ or $K$. But, we do have control over the selection of the centroids $c_{j}$ and the assignment of points to centroids via the function centroid $\left(X_{i}\right)$. The choice of centroids and the assignment of points to centroids will clearly influence the compactness (i.e., "shape") of the resulting clusters.

Let

$$
\text { distortion }=\sum_{i=1}^{n} d\left(X_{i}, \text { centroid }\left(X_{i}\right)\right) .
$$

Intuitively, the smaller the distortion, the better, since a smaller distortion implies that individual clusters are more compact. ${ }^{1}$

For example, consider the data in Figure 1, where the same data points are clustered in two different ways. It is clear that the clustering on the left-hand side in Figure 1 has a smaller distortion than that on the right-hand side. Therefore, we would say that the left-hand clustering is superior, at least with respect to the measure of distortion.

\footnotetext{
${ }^{1}$ In addition to having compact clusters, we might also want a large separation between clusters. However, such separation is not (directly) accounted for in $K$-means.
} 

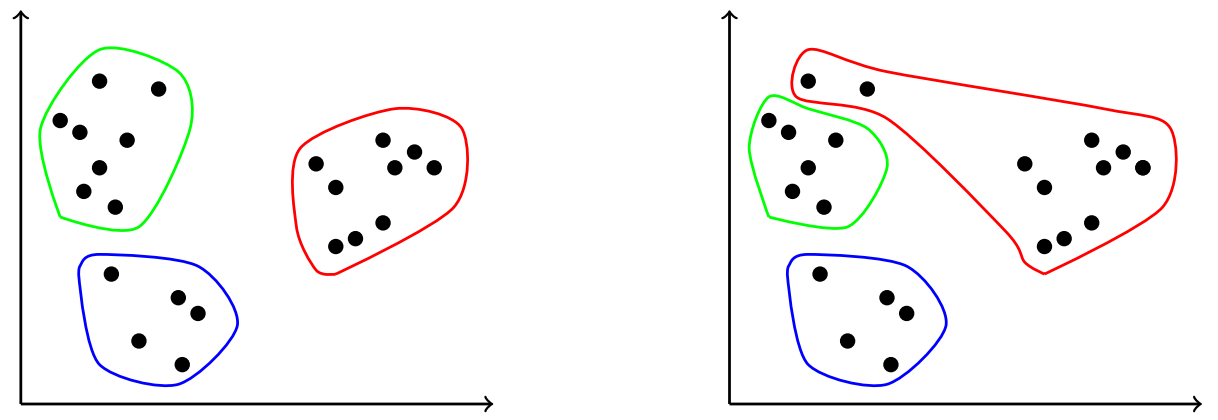

Figure 1: Smaller and larger distortion for the same dataset

Suppose that we try to minimize the distortion. First, we observe that distortion depends on $K$, since more clusters implies more centroids - in the limit, we could let $K=n$, and make each data point a centroid, in which case the distortion is 0 . To emphasize this dependence on $K$, we write distortion $_{K}$. As mentioned above, we assume that $K$ is specified in advance.

The problem we want to solve can be stated precisely as

$$
\begin{aligned}
& \text { Given: } K \text { and data points } X_{1}, X_{2}, \ldots, X_{n} \\
& \text { Minimize: distortion } K=\sum_{i=1}^{n} d\left(X_{i}, \operatorname{centroid}\left(X_{i}\right)\right) .
\end{aligned}
$$

Finding an exact solution to this problem is computationally infeasible. But, there is a simple iterative approximation that works well in practice.

We claim that a solution to (2) must satisfy the following two conditions.

Condition 1 Each $X_{i}$ is clustered according to its nearest centroid. That is, if the data point $X_{i}$ belongs to cluster $C_{j}$, then $d\left(X_{i}, c_{j}\right) \leq d\left(X_{i}, c_{\ell}\right)$ for all $\ell \in\{1,2, \ldots, K\}$, where the $c_{\ell}$ are the centroids.

Condition 2 Each centroid is located at the center of its cluster.

To verify the necessity of Condition 1 , suppose that $X_{i}$ is in cluster $C_{j}$ and that we have $d\left(X_{i}, c_{\ell}\right)<$

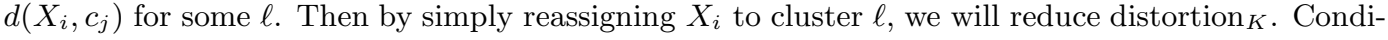
tion 2 also seems intuitively clear, and it is a straightforward calculus exercise to prove the necessity of this condition as well.

Condition 1 tells us that given any clustering for which there are points that are not assigned to their nearest centroid, we can improve the clustering by simply reassigning such data points to their nearest centroid. Condition 2 implies that we always want a centroid to be at the center of its cluster. Therefore, given any clustering, we may improve it - and we cannot make it worse - with respect to distortion ${ }_{K}$ by performing either of the following two steps.

Step 1 Assign each data point to its nearest centroid.

Step 2 Recompute the centroids so that each lies at the center of its respective cluster.

It is clear that nothing can be gained by applying Step 1 more than once in succession, and the same holds true for Step 2. However, by alternating between these two steps, we obtain an iterative process that yields a series of solutions that will generally tend to improve, and can never get worse with respect to distortion ${ }_{K}$. This is precisely the $K$-means algorithm [7], which we state somewhat more precisely in Table 3.

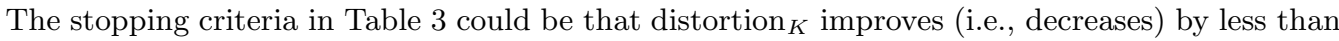
a set threshold, or that the centroids do not change by much, or we could simply run the algorithm for a fixed number of iterations.

Note that the algorithm in Table 3 is a hill climb, and hence $K$-means is only assured of finding a local maximum. And, as with any hill climb, the maximum we obtain will depend on our choice for the initial conditions. For $K$-means, the initial conditions correspond to the initial selection of centroids. Therefore, it can be beneficial to repeat the algorithm multiple times with different initializations of the centroids.

In this experiments below, we use the $K$-means clustering algorithm to explore the natural formation of malware clusters. We employ elbow plots as a tool to discern structure from the 20 malware families based on pairwise clusters. Next, we discuss elbow plots in this context. 


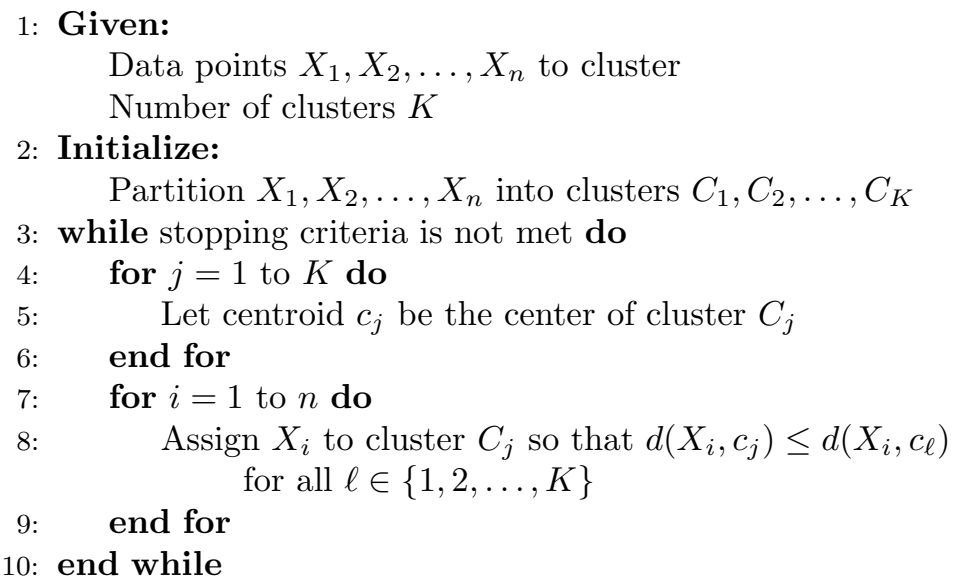

\subsection{Elbow Plots}

Suppose that we graph the clustering error as a function of the number of clusters, $K$. Then an "elbow" in this graph indicates the point where adding another cluster does not significantly improve the clustering results [5]. Such an elbow can be used to determine the (near) optimal number of clusters.

We choose distortion and inertia for our elbow plots. Distortion is calculated as the average of the squared Euclidean distances from each point to the nearest centroid, whereas inertia is calculated as the sum of these same distances. For our experiments, elbow plots using distortion and inertia indicate that the clusters are not well formed, and thus, the number of clusters is somewhat subjective. From Figure 2, it appears that $K \in\{4,5,6\}$ should be good values for the number of clusters, as the inertia and distortion only slightly decrease from that point onward. In any case, these elbow plots clearly indicate that the optimal number of clusters is less than 10, which is somewhat surprising, given that we are dealing with 20 families. This is a strong indication that there is significant similarity between some of the families in our dataset.

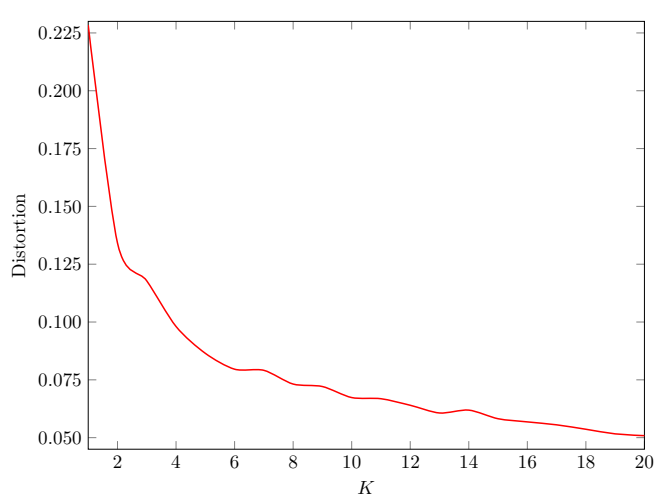

(a) Distortion

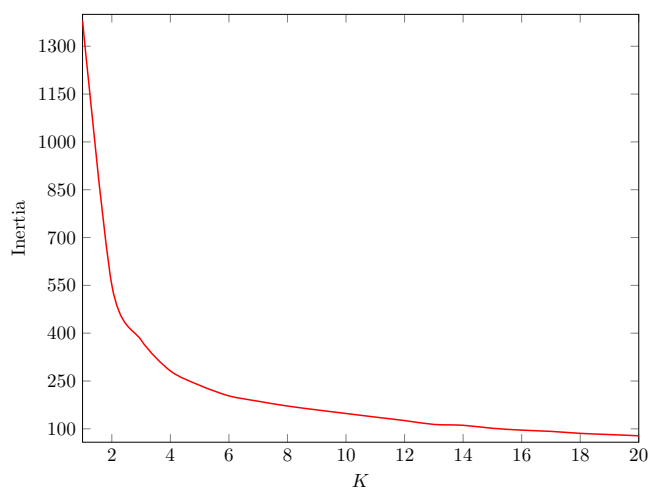

(b) Inertia

Figure 2: Elbow plots

\section{Experiments and Results}

In this section, we present results of three different sets of clustering experiments. First, we cluster each pair of malware families, and show that we can draw meaningful conclusions based on these clustering results. Then we consider clustering experiments where we restrict our attention to one family of each malware type under consideration. Finally, we consider clustering multiple families from the same malware type. 


\subsection{Clustering by Family}

In this set of experiments, we perform clustering for each pair of families. Since there are 20 families, we have $\left(\begin{array}{c}20 \\ 2\end{array}\right)=190$ such clustering experiments. In each case, the top $20 n$-grams is extracted to form the features under consideration. Every sample in the two families under consideration is then converted to a normalized vector of $n$-gram frequencies. The resulting data is clustered using $K$-means, with $K=2$.

The results of these experiments consist of 190 ARI scores and 190 confusion matrices. Representative examples of the resulting confusion matrices are given in Figure 3.

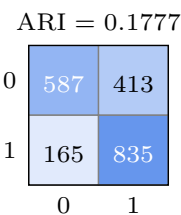

(a) Agent Startpage

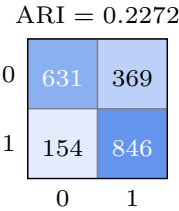

(f) Renos

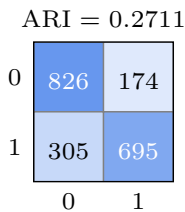

(k) Startpage

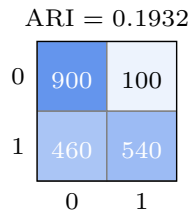

(b) Obfuscator

DelfInject

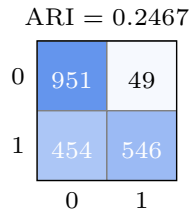

(g) $\begin{aligned} & \text { Alureon } \\ & \text { DelfInject }\end{aligned}$

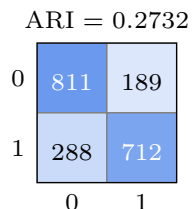

(1) Startpage

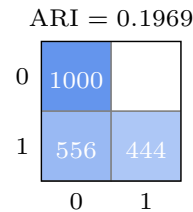

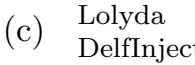

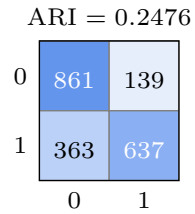

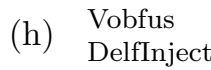

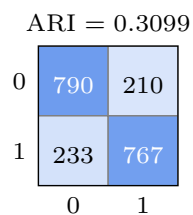

(m) Zeroaccess

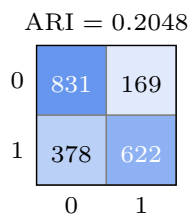

(d) $\mathrm{BHO}$

DelfInject

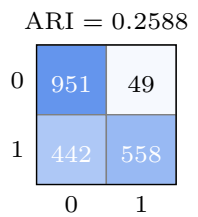

(i) OnLineGames DelfInject

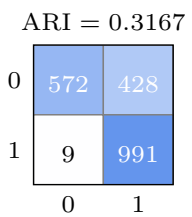

(n) Hotbar

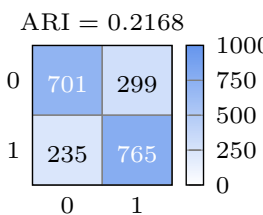

(e) FakeRean

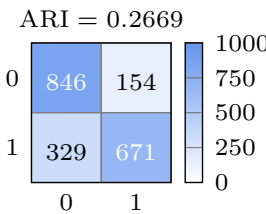

(j) Lolyda

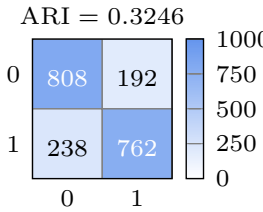

(o) Winwebsec

Figure 3: Selected examples from the 190 pairwise confusion matrices

Each of these 190 clustering experiments provides information on how closely one family is related to another. From such results, we can deduce weak and strong links between malware family pairs. The 190 ARI similarity scores are given in the form of a heatmap in Figure 4. Note that the diagonal elements are 1 in every case, since the similarity between a family and itself is always 1 . Also, the heatmap is symmetric, since the ARI similarity score is itself symmetric.

Figure 4 gives the total pairwise ARI for each family in the form of a bar graph. That is, each bar represents the sum of the 19 ARI scores of a given family with all other families in our dataset. We refer to this sum as the total ARI.

In Figure 5 we give the average ARI for all pairwise clusters formed with a given family. Based on the horizontal line at $y=0.5$, we see that there are four families with a high average ARI, that is, an ARI that exceeds the $y=0.5$ line. This implies that when each of these four families is clustered against the other families, the ARI is, on average, particularly high. The four high-ARI families are BHO, Adload, Hotbar, and Vobfus. Note that these strong ARI results are also apparent from the total ARI scores in Figure 4 and from the heatmap in Figure 4.

From Table 1, we see that Hotbar is the only adware in the dataset and Vobfus is the only worm. It is intuitive that these malware families would tend to stand out more from the other families, due to their being of unique types, and would thus be easier to cluster. This is clearly indicated by the high ARI results for Hotbar and Vobfus. On the other hand, BHO and Adload are both Trojans, which is the most common type in our dataset. This result indicates that in spite of Adload and BHO being Trojans, they contain byte bigram features that are significantly different from the other Trojans in the dataset, namely, Agent, Alureon, Renos, Startpage, Vundo, and Zeroaccess. It is also interesting that Adload and BHO are similar to each other, in the sense that their pairwise clustering result is poor, as can be observed from the heatmap in Figure 4. 
Table 4: Heatmap of pairwise clustering ARI scores
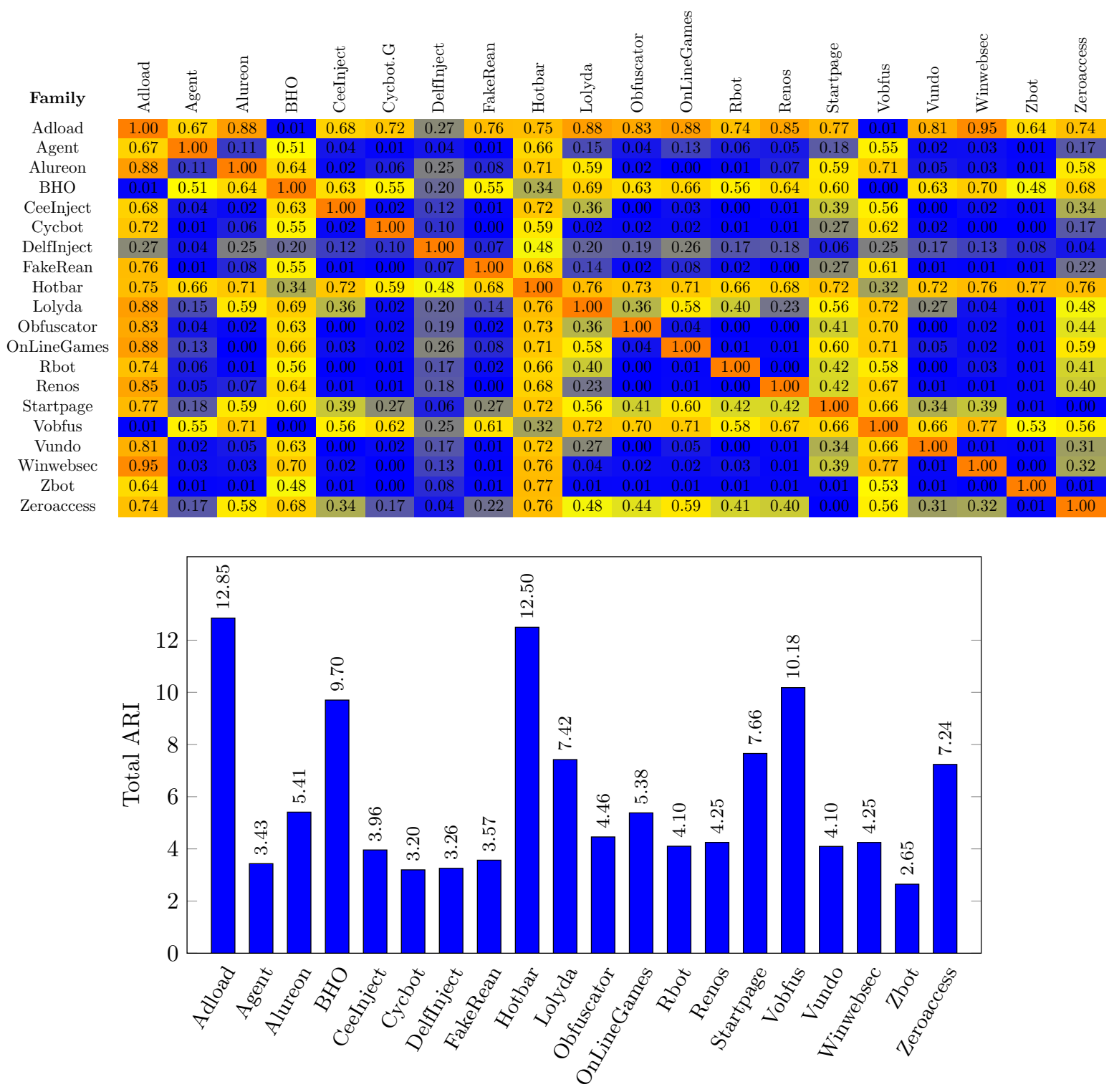

Figure 4: Total ARI score (sum of 19 ARI scores) for each family

To further explore these high ARI families, we provide graphs showing the relationship strength of each with respect to all other families. To generate these graphs, we use the NetworkX library in Python. The resulting graphs are given in Figures 6 through 9, where each node represents a family, with the node numbers corresponding to the "index" column in Table 1. In each of these figures, the darkened node corresponds to the family mentioned in the caption. Also, a dotted edge between two nodes indicates an ARI score of 0.5 or less, while a solid line represents an ARI score greater than 0.5. The nodes are positioned by simulating a force-directed representation, based on the Fruchterman-Reingold force-directed algorithm [6].

These graphs help visualize how other families are related to the four most-distinct families in our dataset. For example, Hotbar is almost equally distinguishable from all other families. On the other hand, Adload is distinguishable from all families except Vobfus and BHO. This means that Adload, BHO, and Vobfus are mostly similar to each other, but highly distinguishable when clustered with other families in the dataset.

It is also interesting to note that there are many families our dataset with extremely poor pairwise clustering results. We see that Agent, CeeInject, Cycbot, DelfInject, FakeRean, Obfuscator, Rbot, Renos, Vundo, Winwebsec, and Zbot all have average ARI scores below 0.23. This indicates that 


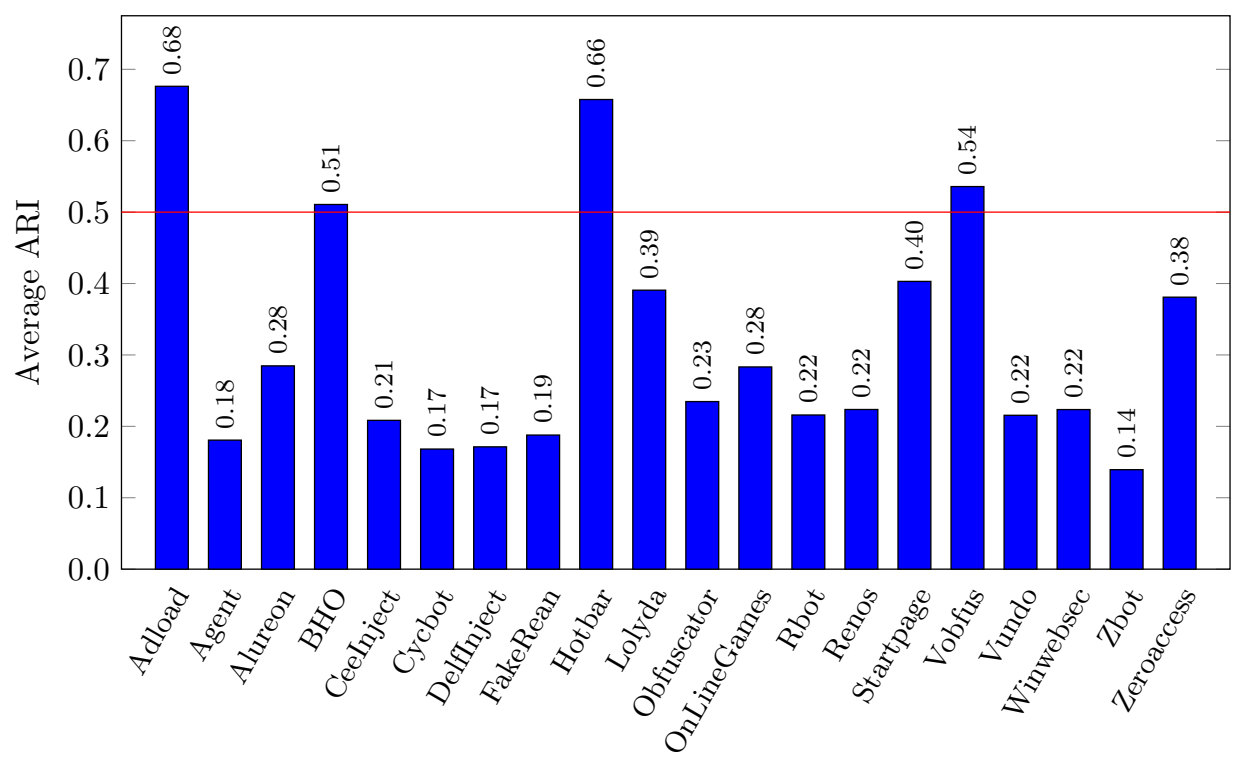

Figure 5: Average ARI score for each family

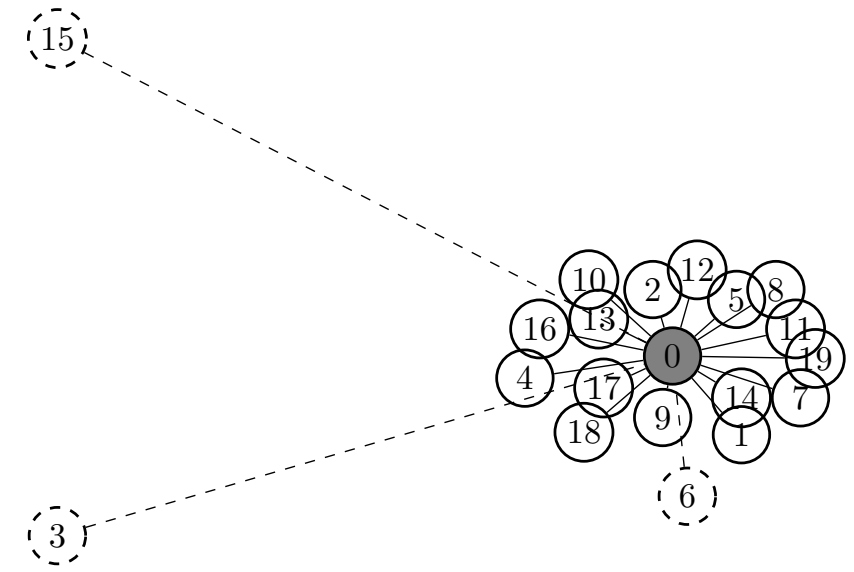

Figure 6: Adload relationship with its paired families

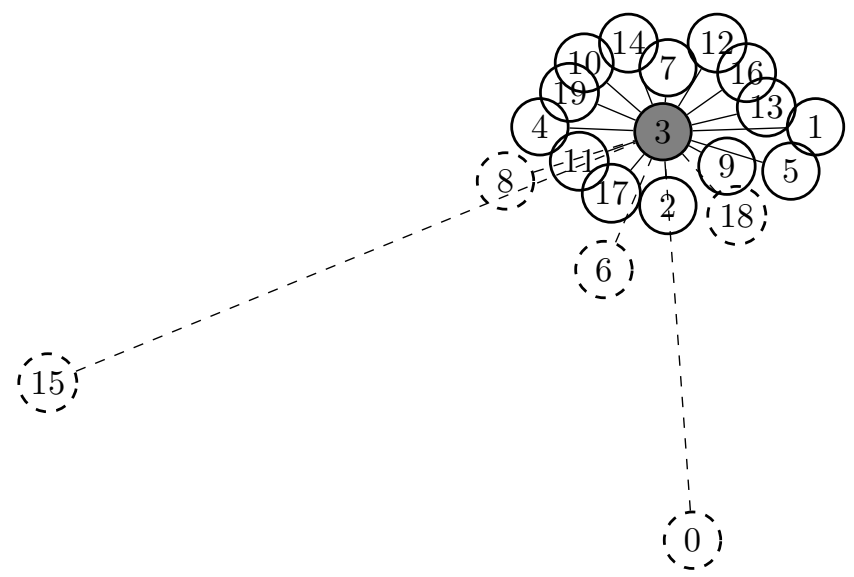

Figure 7: BHO relationship with its paired families

there is a large subset of the families that are virtually indistinguishable from each other. 


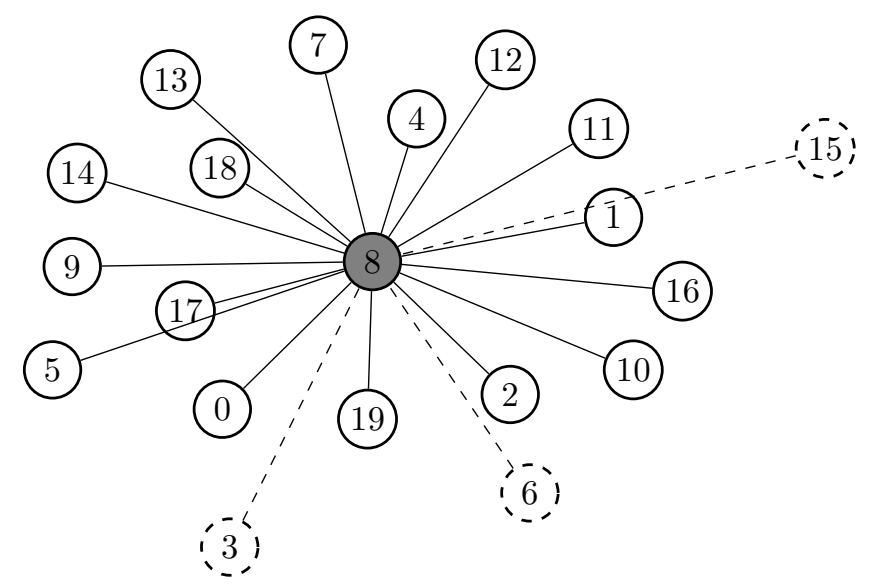

Figure 8: Hotbar relationship with its paired families

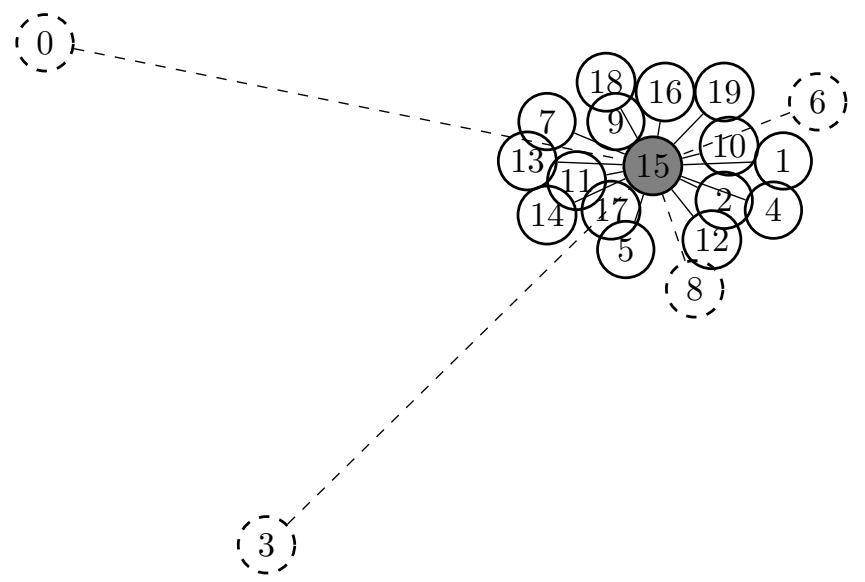

Figure 9: Vobfus relationship with its paired families

\subsection{Clustering Families of Different Type}

In this set of clustering experiments, we consider seven families, each of which is of a different malware type. Specifically, the seven families considered, and their type, are the following:

Agent - Trojan

Ceeinject - VirTool

Cycbot - Backdoor

FakeRean - Rogue

Hotbar - Adware

Lolyda - Password Stealer

Vobfus - Worm

Figure 10 shows the results of clustering these seven families, each of which belongs to a different malware type. We might expect well-defined clusters in this case, but the ARI score is only 0.23 , suggesting that a few families are still very similar, in spite of belonging to different malware types.

The results in Figure 10 indicates that "type" is not a strong feature of malware. More specifically, we can say that the characteristics of bigrams that distinguish one malware family from another are not strongly type-dependent. This somewhat surprising result is useful, since it shows that attempts to identify malware by generic type are, in general, unlikely to be successful, at least when the analysis is based on byte bigram features. However, we note in passing that the authors in [9] use API calls as features and appear to have successfully classified selected malware by its type. Hence, it may be possible to obtain better results for malware type by using other features. 


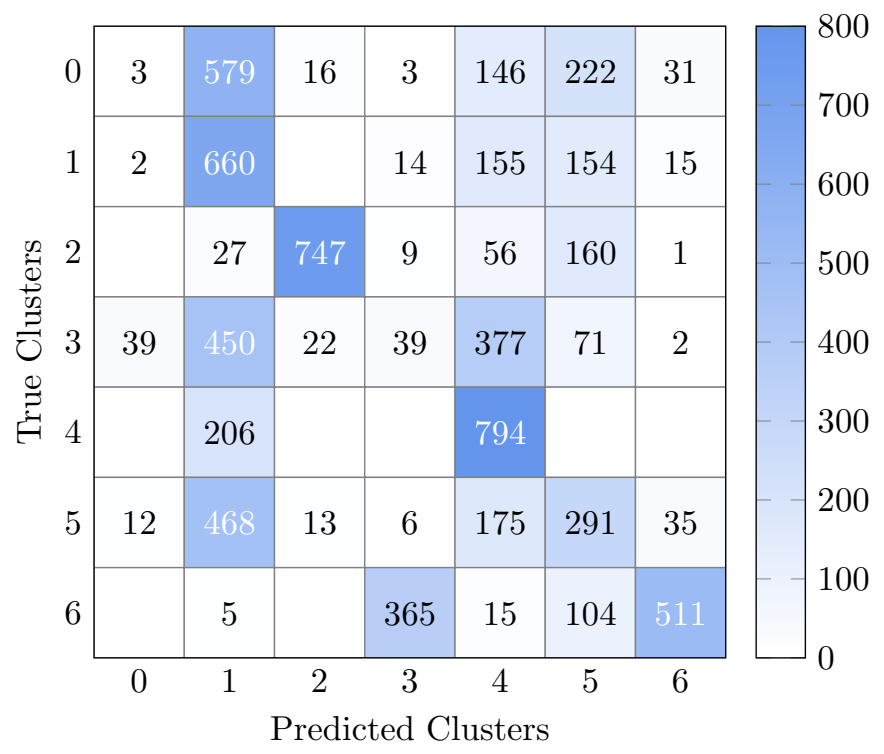

Figure 10: Clustering using seven families from different malware types

\subsection{Clustering Families of the Same Type}

In this section, we conducted two experiments to examine how well $K$-means clustering can distinguish between families belonging to the same malware type. Figure 11 illustrates the results of clustering four families, all of which are Trojans - the specific families considered in this case are Agent, Alureon, BHO, and Startpage. This result suggests that there are three well-defined clusters among these four families. We obtain an ARI of 0.35 in this case, which, interestingly, is much higher than the result obtained for malware samples of different types in the previous section.

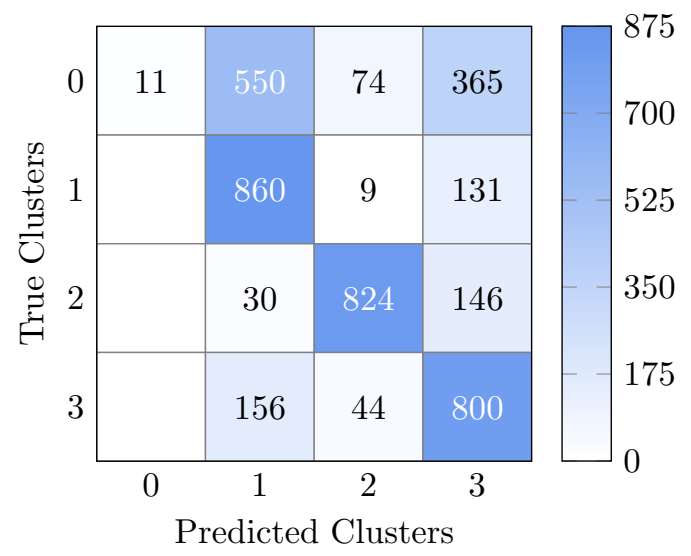

Figure 11: Clustering four Trojan families (Agent, Alureon, BHO, and Startpage)

Next, we cluster the three VirTool families in our dataset, namely, CeeInject, DelfInject, and Obfuscator. In this, we obtain the results in Figure 12, which give us an ARI score of just 0.07. This number suggests a random clustering result, and implies that these VirTool families are virtually indistinguishable, based on byte bigram features.

The results in this section indicate that the Trojan type is generic, in the sense that Trojan families can (and generally do) differ significantly from each other. This is not surprising, as Trojan code tends to be dominated by the non-malicious part of the application, which would be expected to vary widely between different Trojan families.

On the other hand, the VirTool type is highly specific, which results in an inability to distinguish between these families. That is, the VirTool type is relatively homogeneous, making such samples difficult to distinguish from each other, even when they are from different families. 


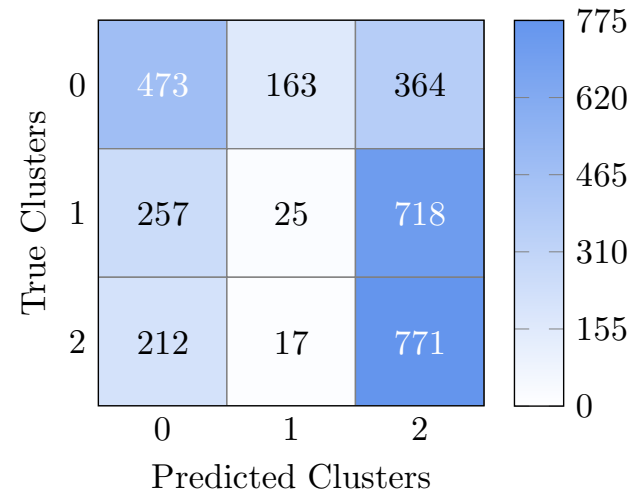

Figure 12: Clustering VirTool families (DelfInject, CeeInject, and Obfuscator)

\section{Conclusion and Future Work}

The goal of this research was to analyze malware clustering, with respect to families and types, based on elementary features and clustering techniques. We considered three different sets of experiments. In our first set of experiments, we clustered all families in pairs. In our second set of experiments, we clustered seven families, with one family from each of the distinct types in our dataset. Finally, we conducted experiments where the clustered families all belong to the same malware type. All of our experiments were based on $K$-means clustering using byte bigram features.

Our findings indicate that the relationship between malware families and malware type is somewhat complex. This is not entirely unexpected, since some malware types, such as Trojans, are only very loosely related, while other types, such as VirTool, are much more specific. Indeed, we did find that families of the Trojan type were far easier to distinguish from each other based on clustering, as compared to VirTool families.

More generally, our pairwise clustering results - and, in particular, the heatmap of ARI scores generated from these pairwise clusters - enabled us to draw many conclusions concerning similarities and differences between families. We were able to clearly see which families were most distinct from all other families, and which subsets of families were the most similar to each other. These results show that elementary cluster analysis is extremely useful for exploring relationships between malware families, and that such analysis could serve as a guide for additional (and more costly) analysis of a given malware dataset.

In this paper, we performed cluster analysis to examine the relationship between malware families. Our focus was clustering using $K$-means and byte bigram features. For future work, it would be interesting to consider larger numbers of clusters, and to explore other clustering techniques, including Gaussian mixture models, hierarchical techniques, spectral clustering, and density-based clustering. While $K$-means can be viewed as generating "circular" or "spherical" clusters, other techniques can produce clusters of more general shapes. In addition, it would be interesting to experiment with other features, such as opcodes and API call sequences.

\section{References}

[1] Adware:win32/hotbar. https://www.microsoft.com/en-us/wdsi/threats/malware-encyclopedia-description?Name=

[2] Backdoor:win32/cycbot.g. https://www.microsoft.com/en-us/wdsi/threats/malware-encyclopedia-description?Nar

[3] Samanvitha Basole, Fabio Di Troia, and Mark Stamp. Multifamily malware models. Journal of Computer Virology and Hacking Techniques, 16(1):79-92, 2020.

[4] Ulrich Bayer, Paolo Milani Comparetti, Clemens Hlauschek, Christopher Kruegel, and Engin Kirda. Scalable, behavior-based malware clustering. In NDSS, volume 9, pages 8-11, 2009.

[5] Purnima Bholowalia and Arvind Kumar. EBK-means: A clustering technique based on elbow method and $k$-means in WSN. International Journal of Computer Applications, 105(9), 2014.

[6] Aric A. Hagberg, Daniel A. Schult, and Pieter J. Swart. Exploring network structure, dynamics, and function using networkx. In Proceedings of the rth Python in Science Conference, SciPy 2008, pages 11-15, 2008. http: //citeseer. ist.psu.edu/viewdoc/download; jsessionid=045872D50E1F472150E79500F79F4B93?doi=10.1.1.522 
[7] Andrew W. Moore. $k$-means and hierarchical clustering. https://www.cs.cmu.edu/ cga/ai-course/kmeans.pdf.

[8] F. Pedregosa, G. Varoquaux, A. Gramfort, V. Michel, B. Thirion, O. Grisel, M. Blondel, P. Prettenhofer, R. Weiss, V. Dubourg, J. Vanderplas, A. Passos, D. Cournapeau, M. Brucher, M. Perrot, and E. Duchesnay. Scikit-learn: Machine learning in Python. Journal of Machine Learning Research, 12:2825-2830, 2011.

[9] Radu S Pirscoveanu, Steven S Hansen, Thor MT Larsen, Matija Stevanovic, Jens Myrup Pedersen, and Alexandre Czech. Analysis of malware behavior: Type classification using machine learning. In 2015 International conference on cyber situational awareness, data analytics and assessment (CyberSA), pages 1-7, 2015.

[10] Gregorio Pitolli, Leonardo Aniello, Giuseppe Laurenza, Leonardo Querzoni, and Roberto Baldoni. Malware family identification with birch clustering. In 2017 International Carnahan Conference on Security Technology, ICCST, pages 1-6, 2017.

[11] Pws:win32/delfinject. https://www.microsoft.com/en-us/wdsi/threats/malware-encyclopedia-description?Name=P

[12] Pws:win32/lolyda.bf. https://www.microsoft.com/en-us/wdsi/threats/malware-encyclopedia-description?Name=PV

[13] Pws:win32/onlinegames. https://www.microsoft.com/en-us/wdsi/threats/malware-encyclopedia-description?Name

[14] Pws:win32/zbot. https://www.microsoft.com/en-us/wdsi/threats/malware-encyclopedia-description?Name=PWS:W

[15] Simone Romano, Nguyen Xuan Vinh, James Bailey, and Karin Verspoor. Adjusting for chance clustering comparison measures. The Journal of Machine Learning Research, 17(1):4635-4666, 2016.

[16] Jorge M Santos and Mark Embrechts. On the use of the adjusted rand index as a metric for evaluating supervised classification. In International conference on artificial neural networks, pages 175-184, 2009.

[17] Matthew Stephens. Dealing with label switching in mixture models. Journal of the Royal Statistical Society: Series B (Statistical Methodology), 62(4):795-809, 2000.

[18] Trojandownloader:win32/adload. https://www.microsoft.com/en-us/wdsi/threats/malware-encyclopedia-descript

[19] Trojandownloader:win32/agent. https://www.microsoft.com/en-us/wdsi/threats/malware-encyclopedia-descripti

[20] Trojandownloader:win32/renos. https://www.microsoft.com/en-us/wdsi/threats/malware-encyclopedia-descriptic

[21] Trojan:win32/bho. https://www.microsoft.com/en-us/wdsi/threats/malware-encyclopedia-description?Name=Tro]

[22] Trojan:win32/startpage. https://www.microsoft.com/en-us/wdsi/threats/malware-encyclopedia-description?Name

[23] Trojan.zeroaccess. https://www.symantec.com/security-center/writeup/2011-071314-0410-99.

[24] Virtool:win32/ceeinject. https://www.microsoft.com/en-us/wdsi/threats/malware-encyclopedia-description?Name

[25] Win32/alureon. https://www.microsoft.com/en-us/wdsi/threats/malware-encyclopedia-description?Name=Win32/

[26] Win32/fakerean. https://www.microsoft.com/en-us/wdsi/threats/malware-encyclopedia-description?Name=Win32

[27] Win32/obfuscator. https://www.microsoft.com/en-us/wdsi/threats/malware-encyclopedia-description?Name=Win3

[28] Win32/rbot. https://www.microsoft.com/en-us/wdsi/threats/malware-encyclopedia-description?Name=Win32/Rbo

[29] Win32/vobfus. https://www.microsoft.com/en-us/wdsi/threats/malware-encyclopedia-description?Name=Win32/V

[30] Win32/vundo. https://www.microsoft.com/en-us/wdsi/threats/malware-encyclopedia-description?Name=Win32/V

[31] Win32/winwebsec. https://www.microsoft.com/en-us/wdsi/threats/malware-encyclopedia-description?Name=Win3 